\title{
Perceptual representation and effectiveness of local figure-ground cues in natural contours
}

\author{
Ko Sakai ${ }^{*}$ Shouhei Matsuoka, Ken Kurematsu and Yasuhiro Hatori \\ Computational Vision Science Laboratory, Department of Computer Science, University of Tsukuba, Tsukuba, Japan
}

OPEN ACCESS

Edited by:

Paul Sajda,

Columbia University, USA

Reviewed by:

Branka Spehar,

The University of New South Wales,

Australia

Shih C. Yen,

National University of Singapore,

Singapore

${ }^{*}$ Correspondence:

Ko Sakai

sakai@cvs.cs.tsukuba.ac.jp

Specialty section:

This article was submitted to

Perception Science,

a section of the journal

Frontiers in Psychology

Received: 15 August 2015

Accepted: 19 October 2015

Published: 03 November 2015

Citation:

Sakai K, Matsuoka S, Kurematsu K and Hatori Y (2015) Perceptual representation and effectiveness

of local figure-ground cues in natural

contours. Front. Psychol. 6:1685.

doi: 10.3389/fpsyg.2015.01685
A contour shape strongly influences the perceptual segregation of a figure from the ground. We investigated the contribution of local contour shape to figure-ground segregation. Although previous studies have reported local contour features that evoke figure-ground perception, they were often image features and not necessarily perceptual features. First, we examined whether contour features, specifically, convexity, closure, and symmetry, underlie the perceptual representation of natural contour shapes. We performed similarity tests between local contours, and examined the contribution of the contour features to the perceptual similarities between the contours. The local contours were sampled from natural contours so that their distribution was uniform in the space composed of the three contour features. This sampling ensured the equal appearance frequency of the factors and a wide variety of contour shapes including those comprised of contradictory factors that induce figure in the opposite directions. This sampling from natural contours is advantageous in order to randomly pickup a variety of contours that satisfy a wide range of cue combinations. Multidimensional scaling analyses showed that the combinations of convexity, closure, and symmetry contribute to perceptual similarity, thus they are perceptual quantities. Second, we examined whether the three features contribute to local figure-ground perception. We performed psychophysical experiments to judge the direction of the figure along the local contours, and examined the contribution of the features to the figure-ground judgment. Multiple linear regression analyses showed that closure was a significant factor, but that convexity and symmetry were not. These results indicate that closure is dominant in the local figure-ground perception with natural contours when the other cues coexist with equal probability including contradictory cases.

Keywords: perception, Gestalt factor, contour shape, natural image, border ownership, psychophysical experiment

\section{INTRODUCTION}

The visual system segregates a scene into regions and assigns figure and ground to them. The shape of the region boundary strongly influences the figure-ground segregation. A number of contour features, such as convexity, closure, symmetry, good continuation, similarity, and proximity, are cues for the segregation (e.g., Brunswik and Kamiya, 1953; Kanizsa, 1979). For instance, psychophysical studies reported a decrease in the detection threshold (e.g., Kovács, 1996) and a decrease in the response time (Elder and Zucker, 1998) for collinearly aligned patches and closed 
boundaries, respectively. A wide range of saliency that depends on symmetry has also been reported (Rainville and Kingdom, 2000; Cohen and Zaidi, 2013). Functional MRI studies have reported the selective responses to colinear contours in the early (V1 and V2) to higher (lateral occipital complex) visual cortical areas (Kourtzi et al., 2003), and the responses to symmetry that were highly correlated with human perception in the intermediate (V3A, V4, and V7) to higher (lateral occipital) areas (Sasaki et al., 2005). Combinations of plural cues have also been studied (Devinck and Spillmann, 2013; Froyen et al., 2013; Mojica and Peterson, 2014). Although contours in natural scenes usually constitute multiple cues, previous studies have focused on testing stimuli in which each cue was independently provided with simplified and artificial topographies.

How coexisting multiple cues contributes to figure-ground perception and how conflicting cues fuse to produce coherent perception have not been clarified. Analyses of the perception of natural contours appear to be ingenious for answering these questions. Within local patches from natural contours, convexity, vertical location, and size provide clues for figureground segregation, with convex, lower, and smaller regions tending to be associated with figures (e.g., Fowlkes et al., 2007; Burge et al., 2010). Combining two or three of these cues further facilitates the perception of figures. Although these studies provided insightful evidence for coexisting cues in natural contours, the factors were image features, and not necessarily perceptual quantities that represent the contours in the visual system. Electrophysiological studies have reported evidence of the cortical representation of angles and curvatures (Pasupathy and Connor, 1999; Ito and Komatsu, 2004), but not of other contour features such as convexity, closure, symmetry, etc. It is crucial to investigate whether these factors are indeed perceptual quantities that represent natural contours. The results of Fowlkes et al. (2007) could be biased because of the natural distribution of the appearance frequency of the factors. More frequently appearing factors could be judged more dominant than those appearing less frequently. The natural distribution could be biased so that contradictory cues barely coexist. It is desirable to annul the frequency of appearance of the factors when examining the effectiveness of coexisting factors. Investigations that focus on perceptual quantities using controlled natural contours are expected to provide crucial evidence for understanding figureground perception.

Figure-ground segregation is often considered as global processing that needs top-down processing. However, local bottom-up processing that is fast and autonomous should also contribute to the segregation before and recurrently with the top-down processing. For instance, local figure-ground assignment could differ from the global perception, depending on instruction, window size, or direction of gaze (Fowlkes et al., 2007; Kim and Feldman, 2009). Other psychophysical studies have reported that figure-ground segregation can occur without focal attention near the point of fixation (Kimchi and Peterson, 2008), and that human development shows differences in detecting local and global configurations (Kimchi et al., 2005). Physiological studies have reported that early- to intermediatelevel visual areas respond to grouping and figure-ground segregation (e.g., Altmann et al., 2003; Tyler et al., 2005). A recent physiological study reported that figure-ground processing in the primary visual cortex (V1) follows each fixation saccade, indicating autonomous bottom-up processing of figure-ground segregation in an early stage (Gilad et al., 2014). Physiological studies of the cortical area (V2) have found a short latency of 20$50 \mathrm{~ms}$ for border-ownership-selective cells (e.g., Zhou et al., 2000; Zhang and von der Heydt, 2010). Although the origin of this latency is controversial, the contribution of bottom-up signals is plausible (Sakai and Nishimura, 2006; Super et al., 2010; Sakai et al., 2012; Sakai and Michii, 2013). Local, autonomous bottomup processing appears to play a crucial role in figure-ground segregation.

We investigated whether the Gestalt factors, specifically convexity, closure, and symmetry, that coexist inherently in natural contours are perceptual quantities that represent local contours. We focused on these three factors as the first step because they appear to represent well the shape of a local contour segment. Specifically, we performed similarity tests between a variety of natural contour patches that were labeled by these three factors, and examined the contribution of the three factors to the perceptual similarity between the contours. The contour patches were sampled so that their distribution was uniform within the space composed of the three contour features. This sampling ensured the equal appearance frequency of the factors and a wide variety of contour shapes including those with contradictory factors that induce the figure in the opposite directions. Because the contradictory cases appear much less frequently in natural distribution, our sampling with uniform distribution is crucial for understanding the cue combinations. We used natural contours as a way to randomly choose a variety of contours that satisfy a wide range of cue combinations, but not to reflect the probability of natural appearance. Multidimensional scaling (MDS) analyses showed that combinations of convexity, closure, and symmetry contribute to the similarities of the natural contours and are indeed perceptual quantities.

Next, we examined whether convexity, closure, and symmetry contribute to local figure-ground segregation in the natural contours when they coexist with nearly equal probability. We performed psychophysical experiments to judge the direction of figure along the contours and examined the contribution of these factors to the figure-ground judgment. A multiple linear regression analysis (MLRA) indicated that closure was a significant factor but convexity and symmetry were not. This result indicates that closure is a stronger cue compared with convexity and symmetry for the figure-ground segregation in the local natural contours, which appears to be natural since convexity represents a part of closed contour. Because the degree of closure and convexity/concavity as well as their appearance frequency were controlled so that they distributed uniformly, these cues were contradictory in about a half of the stimuli. Our result suggests the dominance of closure over convexity when they are contradictory (see Figure $\mathbf{1 C}$ for examples). These results indicate that convexity, closure, and symmetry are perceptual quantities that represent local contours, and that closure is dominant among them when these factors coexist with equal probability including contradictory cases. 


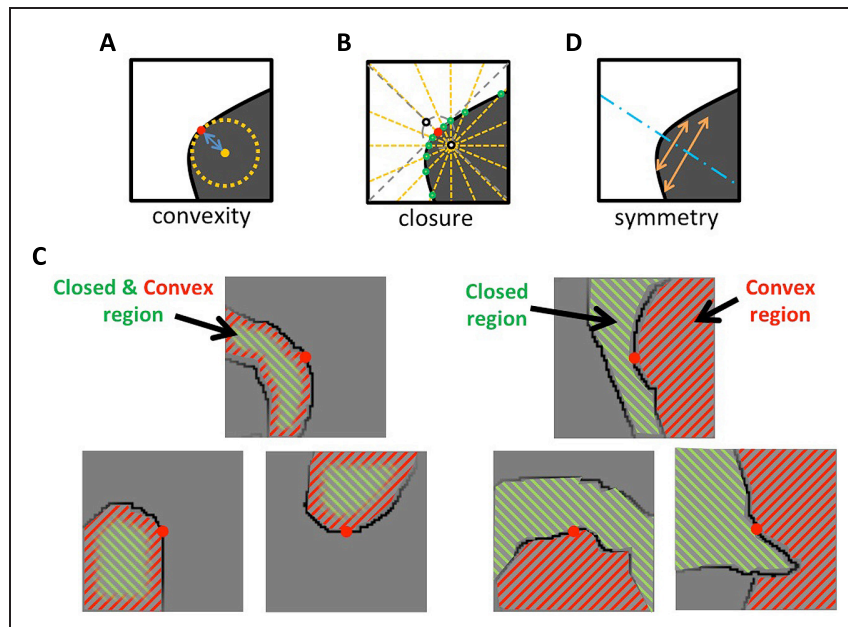

FIGURE 1 | Illustrations for the quantification of the three Gestalt cues. (A) Convexity was defined as the curvature around the center of a patch. (B) Closure was defined from the number of radial lines that cross the contours. The degree of Closure is positive if a contour is closed more on the convex side, and negative if closed on the concave side. (C) The left panels show examples that Closure is positive where the convex side is closed more than the concave side $\left(N_{\text {convex }}>N_{\text {concave }}\right)$. In this case, convexity and closure draw figure-ground perception in the same direction. The right panels show examples that Closure is negative where the concave side is more closed. In this case, convexity and closure conflict with each other. (D) Symmetry was defined as the degree of overlap between the contours of both sides with respect to the symmetry axis. The blue dotted line shows the optimal symmetry axis. See the text for details.

\section{MATERIALS AND METHODS}

To analyze the contribution of contour shape to the perception of similarity and local figure-ground segregation, we quantified contours with local cues, specifically, convexity, closure, and symmetry. In the present study, we used the standard definitions for convexity and closure (e.g., Sajda and Finkel, 1995; Fowlkes et al., 2007). Convexity was defined by curvature, and closure was defined by the number of radial lines that crossed the contour, as is shown in detail in the following subsections. Symmetry was defined as a quantitative measure that describes how close a local contour is to the axial symmetry (Sakai et al., 2014). We divided a number of natural contours into local patches, labeled them with the three measures, and sampled stimuli from them. We endeavored to sample patches so that they distributed uniformly over the space that was constituted of the three measures. This sampling resulted in a wide variety of contour shapes with a limited number of patches, including those with contradictory factors that induce figure in the opposite directions. Note that we used natural contours as a way to randomly pickup a variety of contours that satisfy particular cue combinations, but not to reflect natural appearance probability. However, the practical limitation on the number of the patches and the property of the natural distribution prevented us from collecting a complete set of patches with uniform distribution. Therefore, we sampled the patches uniformly from the space constituted of convexity and closure, and confirmed that symmetry of the chosen set was widely distributed. We used this set of local contours for the present experiments, with each patch labeled by the degree of convexity, closure, and symmetry. The experiments were approved by the Research Ethics Committee of the Institute of Systems and Information Engineering, University of Tsukuba, in accordance with the Code of Ethics of the World Medical Association (Declaration of Helsinki). Informed consent was obtained for experimentation with the participants.

\section{Definitions of Convexity, Closure, and Symmetry}

We selected squares of $69 \times 69$ pixel from a set of contours that contained human-marked segmentation of natural scenes (Berkeley Figure/Ground Dataset, BFGD; Martin et al., 2001) with the center of a square always placed on the contour. The shape of the local contours was quantified with degrees of convexity, closure, and symmetry, as detailed below.

\section{Convexity}

We used the standard definition for convexity (Fowlkes et al., 2007) as defined by:

$$
\operatorname{Convexity}\left(x_{u}, y_{u}\right)=\log \left(1+\left|\rho\left(x_{u}, y_{u}\right)\right|\right)
$$

where $\rho$ represents the curvature at the center of a patch $\left(x_{u}\right.$, $y_{u}$ ). We took into account 41 pixels along the contour for the computation of $\rho$, with the midpoint at the center of a patch $(\rho$ represents the curvature of a contour segment with the length of 41pixels). An illustration of Convexity is shown in Figure 1A. Convexity is always positive. The opposite of the convex side was defined as the concave side. Examples of patches with particular values of Convexity are shown in Figure 2.

\section{Closure}

The closure index, used in the present experiment, was defined by Sajda and Finkel (1995). In brief, closure was defined as the number of radial lines that crossed the contour in a square patch, as illustrated in Figure 1B. We considered the normal of the contour that passed through the center of a patch $\left(x_{u}, y_{u}\right)$. We chose two points along the contour normal that were equidistant (5 pixel) from the center (black open circles in Figure 1B), and drew 16 radial lines with an increment of $22.5^{\circ}$ from each of the two points. We counted the number of lines that crossed the contour for each point. Closure of a patch was defined as the difference between the number of crossing lines in convex side, $N_{\text {convex, }}$ and that in concave side, $N_{\text {concave }}$ :

$$
\text { Closure }\left(x_{u}, y_{u}\right)=N_{\text {convex }}-N_{\text {concave }}
$$

Closure is positive if the closed side corresponds with the convex side, and negative if it corresponds with the concave side. Examples of positive and negative cases are illustrated in Figure 1C. Examples of patches with particular values of Closure are shown in Figure 2.

\section{Symmetry}

We proposed the degree of symmetry as a quantitative measure to describe how close a local contour is to the axial symmetry (Sakai 


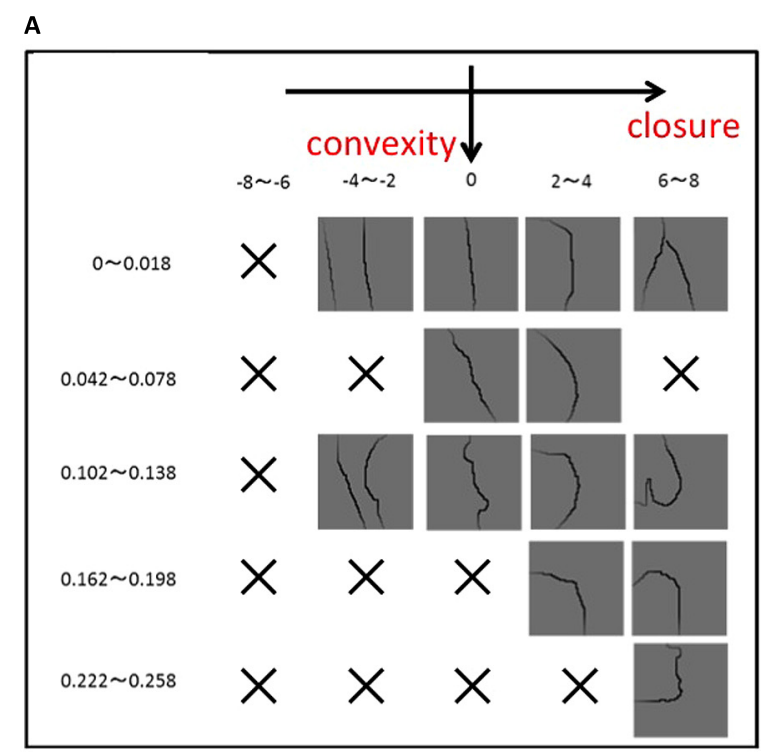

B

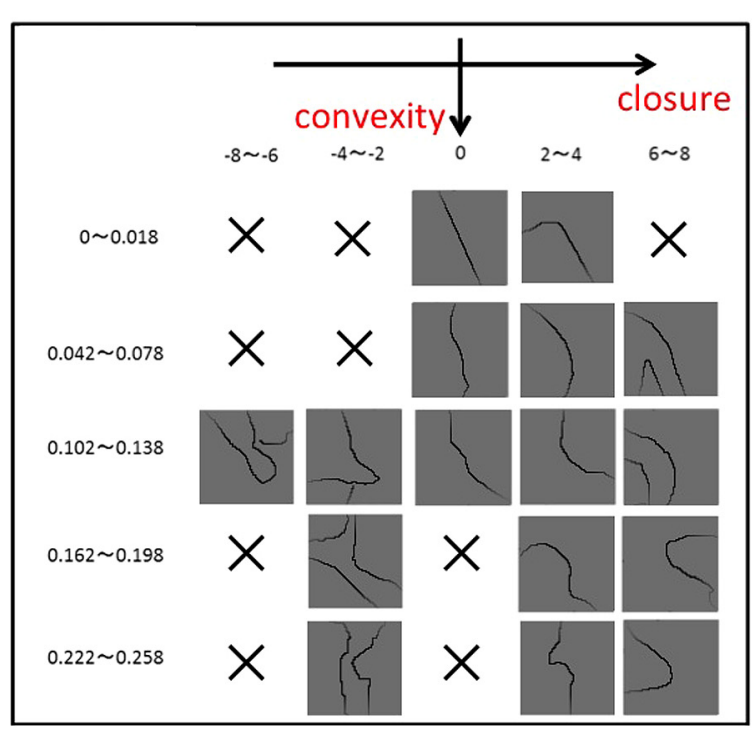

FIGURE 2 | Examples of the stimuli used in the experiments. To ensure a wide variety of contour shape, we classified patches based on the degree of Convexity, Closure, and Symmetry, and sampled them as uniformly as possible. (A) The patches in which the contour tangent at the center ranges between $0^{\circ}$ (vertical) and $-15^{\circ}$ (counterclockwise), and $\mathbf{( B )}$ between $-15^{\circ}$ and $-30^{\circ}$. The degree of Convexity increases from the top $(0-0.018)$ to the bottom (0.222-0.258), and Closure from the left (-8 to -6$)$ to the right (6-8). There are a number of the patches in which Convexity and Closure correlate positively, but there are few or no patches with the negative correlation. Specifically, patches with a large Convexity and a negative Closure, as examples shown in Figure 1C, barely exist in the Berkeley Figure/Ground Dataset (BFGD).

et al., 2014). Because contours are barely symmetric in terms of geometry, a measure to describe the degree of symmetry needs to be defined. The degree of symmetry was defined as the degree of the overlap of the contours between the two sides divided by the optimal axis of symmetry, as illustrated in Figure 1D. The optimal axis provides the maximum overlap of contours between the two sides, which was searched thoroughly by rotating $(\theta)$ and translating $(x)$ the patch with respect to the vertical at the center. The overlap of the contours between the two sides ( $a$ and $b$ ) was given by:

$$
\operatorname{dos}_{\theta, x}=\frac{\sum_{i=1}^{N}\left(\sum_{j=1}^{N}\left(a_{i j} b_{(N-i+1), j}\right)\right)}{\text { length }}
$$

where $i$ and $j$ correspond to the $x$ and $y$ directions of the rotated/translated patch, respectively, with the axis of symmetry set to the vertical at the center and the origin at the top-left corner. $N$ is the spatial extent of the patch in pixel $(N=69)$. The overlap was normalized by the length of contour in the patch (length). We normalized dos by the largest dos among all patches $(m)$ taken from BFGD, and defined it as Symmetry for the patch $k$ :

$$
\text { Symmetry }_{k}=\frac{\max _{\theta, x}\left(\operatorname{dos}_{k, \theta, x}\right)}{\max _{m, \theta, x}\left(\operatorname{dos}_{m, \theta, x}\right)}
$$

This normalization assures the independence of Symmetry from patch size (Convexity and Closure are independent, but not dos). We confirmed that the patch with the largest dos was perfectly symmetric, therefore, the patch with Symmetry $=1$ shows perfect symmetry. The sign of Symmetry was set negative if the length of the optimal axis passed through the convex side was shorter than that through the opposite side. This sign setting establishes the consistency among convexity, closure, and symmetry, in terms of which side tends to be figure (the positive sides tend to be figure). For instance, if a region is convex and closed, and the symmetry axis passes through it, all the cues indicate consistently that this region is likely to be figure. By contrast, if the symmetry axis is located outside of the convex region, the convex and symmetry cues conflict with each other; convex draws the perception of the figure toward the inside of the convex region and symmetry toward the outside. In this case, the indices should have the opposite signs to clarify the direction that the index draws the figural perception.

\section{Stimulus Selection and Presentation}

Based on the three indices, Convex, Closure, and Symmetry, we selected 105 patches from more than 10,000 patches taken from BFGD. Before the selection, we excluded patches that match at least one of the following conditions: (1) contours crossed each other (X-junction), (2) a whole object (e.g., human, animal, or flower) was visible, or (3) contours were packed (complicated contours were closely located to each other) so that it was difficult to assess the direction of figure. The second and third conditions were tested by the visual inspection of three people (two were the authors) who were familiar with the dataset and did not participate in the experiments.

To assure a wide variety of contours, we classified the patches based on Convex, Closure, Symmetry, and the orientation (tangent) of the contour passing through the patch center. The contour was classified into eight orientations $\left(0-15^{\circ}, 15-30^{\circ}\right.$, $30-60^{\circ}, 60-90^{\circ}, 90-120^{\circ}, 120-150^{\circ}, 150-165^{\circ}$, and $165-180^{\circ}$; $0^{\circ}=$ vertical). Convex and Closure were categorized into five classes, respectively, as indicated in Figure 2. The patches 
were grouped into 200 classes that consisted of five classes for Convexity, five for Closure, and eight for orientation. One patch was chosen randomly from each class. The limited number of patches and the natural distribution of contour shapes made it impossible to fill all the classes. We performed a preliminary experiment to test whether the rank orders of Convexity and Closure agreed with those of perception, and chose 105 patches that showed agreement. The details of this procedure are described in Appendix A in the Supplementary Material. The selected set of stimuli is shown in Appendix B in the Supplementary Material. We confirmed that Symmetry of the chosen patches was widely distributed. The distribution of the patches as a function of the factors is shown in Appendix $\mathrm{C}$ in the Supplementary Material. Although our measure of Symmetry reflects the perception in some degree, the agreement between the two was less accurate than those for convexity and closure, and the details are described in Appendix A in the Supplementary Material. Note that we were unable to obtain a complete set of patches with the uniform distribution of the three factors because of the practical limitation on the number of patches and the natural distribution of contour shapes. To smooth patch boundaries, we multiplied the patches with a Gaussian ( $\sigma=17$ pixel). Examples of the patches are shown in Figure 2. We presented the patches on a liquid crystal display (Mitsubishi RDT197S) that was placed in a dark room. Contours were presented in black $\left(0.86 \mathrm{~cd} / \mathrm{m}^{2}\right)$ on a gray background $\left(62 \mathrm{~cd} / \mathrm{m}^{2}\right)$. A fixation aid was presented in $\mathrm{red}\left(72 \mathrm{~cd} / \mathrm{m}^{2}\right)$.

\section{PERCEPTUAL SIMILARITY OF LOCAL CONTOURS - EXPERIMENT 1}

We investigated whether convexity, closure, and symmetry are indeed perceptual quantities to represent the perception of contour shape. Although these factors are widely known as Gestalt factors for grouping and figure-ground organization, it is uncertain whether the factors are the perceptual quantities that represent the contour shape in natural scenes. We determined psychophysically the multidimensional configuration that represents the perceptual shape of local contour, and analyzed whether these factors could be the configuration axes. Specifically, we performed similarity tests between a wide variety of natural contour patches in which multiple cues coexist, and performed MDS analyses. We generated the multidimensional configuration that represented the perceptual distance of local contour shapes from the similarity tests, and examined whether this arrangement agreed with the configuration that was composed of Convexity, Closure, and Symmetry. If the result showed agreement, it suggests that these factors are indeed perceptual quantities in the judgment of the shape of natural contours.

\section{Procedure}

We performed similarity tests between the natural contours that were labeled with Convexity, Closure, and Symmetry. To ensure a practical duration of the experiment, we limited the number of stimulus to 54 out of 105 (the selected set; see Materials and Methods). Specifically, we used the stimuli in which the contour tangent ranges between $0^{\circ}-30^{\circ}$ and $150^{\circ}-180^{\circ}$ with respect to the vertical. The experimental procedure is shown in Figure 3. Two stimulus patches of $4^{\circ} \times 4^{\circ}$ in visual angle were presented side-by-side with the interval of $1^{\circ}$ at the center of the display, following the presentation of a mask for $1500 \mathrm{~ms}$. A fixation aid of a small red square was presented with the stimuli and the mask. Participants were asked to judge the similarity of the shape of the local contours in the right and left patches as soon as possible. Specifically, they were instructed to rate the similarity with five scales (subjective rating method) by choosing one key out of five. Immediately after the answer, the next mask was presented. Pairs of stimuli $(n=1431)$ were presented in random order with four repetitions. Six participants aged in their twenties (21-28) with normal or corrected-to-normal vision underwent the experiment. Before the experiment, the participants were familiarized with the task by performing the same procedure but with a small, different set of patches chosen exclusively from the selected set. During the familiarization, the participants were asked to categorize the patches evenly to five scales.

\section{Results}

We performed tests between natural contours to determine the perceptual similarity among the contours. MDS was applied to generate the perceptual configuration of the contours that represents the similarity among patches by the distance between the points corresponding to the patches in a low-dimensional space (e.g., Dillon and Goldstein, 1984). In other words, perceptually similar contours were placed close to each other while dissimilar contours were placed separately at a distance in a space with one to three dimensions (1D to $3 \mathrm{D}$ ). With this configuration, the rank order of perceptual similarity should agree with that of proximity. However, some patches violated this relation because the dimension of the configuration space was far lower than that of the patches. This error was evaluated by the stress as defined by Kruscal (1964) that takes zero for a perfect

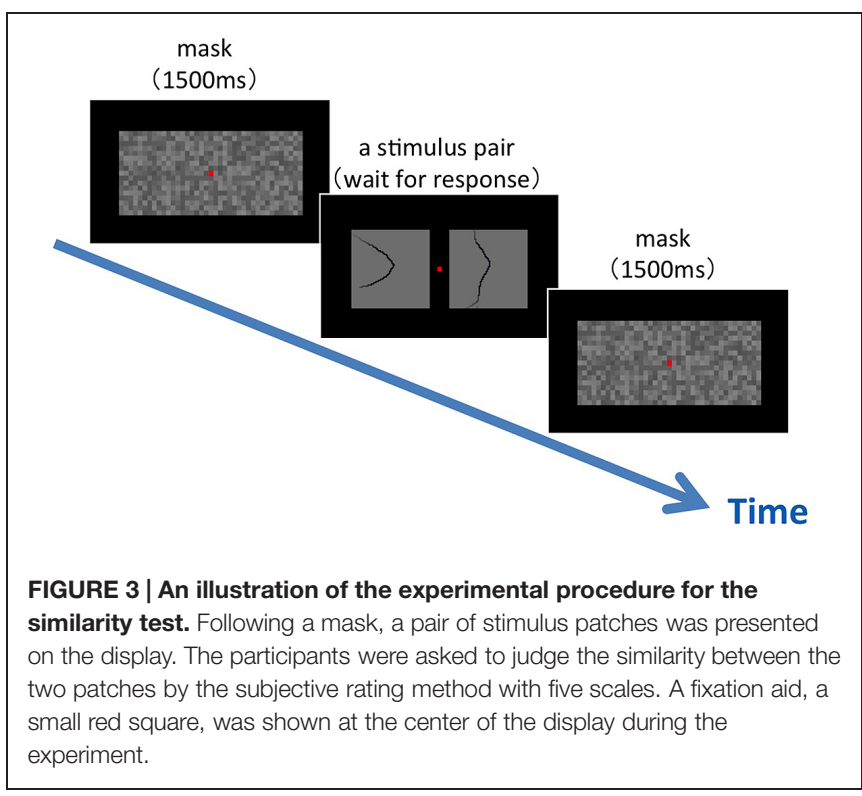


match between the rank orders and one for no match. The stress is defined as follows:

$$
\text { stress }=\frac{\sum \sum\left[\theta\left(d_{i j}\right)-\hat{d}_{i j}\right]^{2}}{\sum \sum \hat{d}_{i j}^{2}}
$$

where $\theta$ is a monotonically increasing function (rank order), and $d$ and $\hat{d}$ represent the Euclidian distances between stimuli $i$ and $j$ in perceptual and topographical configurations, respectively. We applied the Kruscal (1964) method (isoMDS in R language for statistical computing) to compute the stress in which the absolute value of the similarity rating was ignored. The mean stresses for 1D, 2D, and 3D configurations among all participants were 43, 24 , and $18 \%$, respectively. The details are shown in Appendix $\mathrm{D}$ in the Supplementary Material. This result suggests that the correctness for the $1 \mathrm{D}$ configuration in the representation of perceptual similarity is about half of the perfect, and that the correctness increases to more than $80 \%$ for the $3 \mathrm{D}$ configuration. These stress values indicate the limitation of the analysis by reducing the dimension. Specifically, 1D, 2D, and 3D analyses explain no more than 57,76 , and $82 \%$ of the perceptual similarity, respectively.

We examined whether topographical measures, Convexity, Closure, and Symmetry, account for the perceptual configuration. Specifically, we tested whether the perceptual configuration of the contours agrees with the topographical configuration of the stimuli. In other words, we generated topographical configurations of the contours by mapping the patches into $1 \mathrm{D}$ to $3 \mathrm{D}$ spaces as defined by the topographical measures and compared them with the perceptual configurations of the same dimension. If the perceptual and topographical configurations show agreement, it suggests that the topographical factors are perceptual quantities in the judgment of similarity between the contours. The agreement further suggests that Convexity, Closure, and Symmetry account for the perception of the contours.

Before the comparison of the perceptual and topographical configurations, we processed the configurations so as to minimize the differences in arrangement between the configurations (Borg and Groenen, 1997). Specifically, we scaled and rotated the configurations using the Procrustes method (Hurley and Cattell, 1962; procrustes function in Vegan package of R) to obtain the best fit (the minimum difference). An example of the configurations of perception and topography with best fit (Convexity and Closure) in $2 \mathrm{D}$ is shown in Figure 4. The $2 \mathrm{D}$ perceptual configuration has two axes, which means that contour shapes are labeled with two factors. Here, we examined whether the contour shape is represented perceptually by convexity and closure. Some patches are located close to each other between the two configurations (the same numbers in red and blue in Figure 4; e.g., Nos. 1, 29, and 40), while others are distant (e.g., Nos. 5, 23, and 42).

To test the significance of the agreement between the perceptual and topographical configurations, we used the squared sum of the pair-wise Euclidian distance of the patches between the configurations (Borg and Groenen, 1997). We defined this squared-sum of distance as Error. To enable intuitive comparison, we normalized the Error by the squared-sum of the distance between the perceptual and random configurations (Error for random configurations). The random configurations were computed repeatedly 100 times by scrambling the similarity among the patches. For the

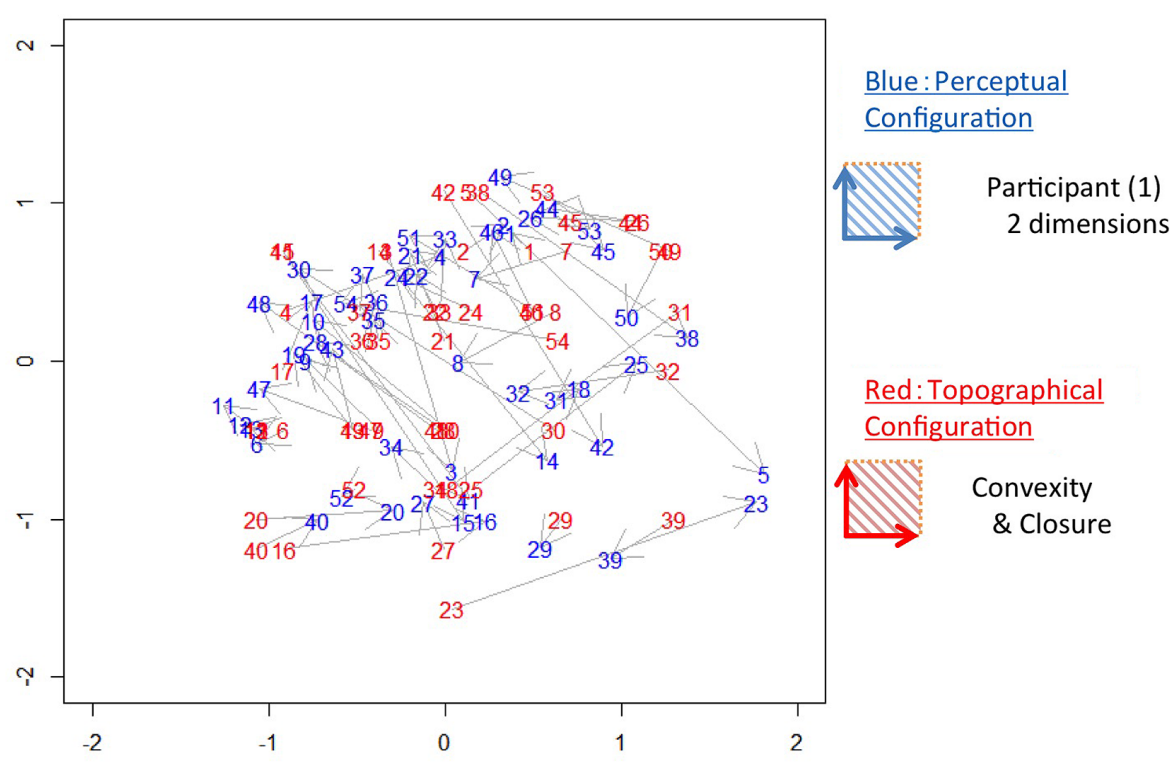

FIGURE 4 | An example of the perceptual and topographical configurations. Numbers in blue (dark gray) represent stimuli in the 2D perceptual configuration in which shorter distances between stimuli indicate greater perceptual similarity. Numbers in red (light gray) represent stimuli in the topographical configuration of Convexity and Closure. The distances between the corresponding stimuli (the same numbers in red and blue) indicate the similarity between the perceptual and topographical configurations. 
example shown in Figure 4, the mean Error among all participants was 0.57 , indicating that Convexity and Closure account for $43 \%$ of the perceptual similarity. A statistical test showed that the Error was significantly smaller than that for the random configurations ( $t$-test, $p<0.001$ ). This result indicates an agreement between the perceptual and topographical configurations, suggesting that Convexity and Closure contribute to the perceptual similarity between natural contours.

We performed the analyses for $1 \mathrm{D}$ to $3 \mathrm{D}$ configurations in which Convexity, Closure, and Symmetry were topographical factors. The computed Errors and their statistical tests are summarized in Figure 5 and Table 1, respectively, and their details are shown in Appendix D in the Supplementary Material. For the comparison of $1 \mathrm{D}$ configurations between the perceptual and topographical configurations, five of six participants found significantly smaller Error than that for the random configurations ( $t$-test, $p<0.05$ ) for Closure, and three for Convexity or Symmetry. The mean Errors among participants for Closure, Convexity, and Symmetry were 68, 74, and 89\%, respectively. These results indicate that Closure, Convexity, and Symmetry account for about $10-30 \%$ of the similarity. The contribution of Closure was significantly larger than Symmetry but not than Convexity (pair-wise $t$-test; $p=0.01$ and 0.3 , respectively). The contribution of Closure and Convexity are surprisingly high. Because the stress of $43 \%$ indicates that $1 \mathrm{D}$ configuration represents no more than $57 \%$ of the perceptual similarity, Closure and Convexity account for about a half of the similarity.

For the $2 \mathrm{D}$ and $3 \mathrm{D}$ configurations, all six participants found significantly smaller Error than that for the random configurations ( $t$-test, $p<0.0013$ ) for all combinations of factors, indicating that these factors contribute to the perceptual similarity between natural contours. For 2D configurations, the

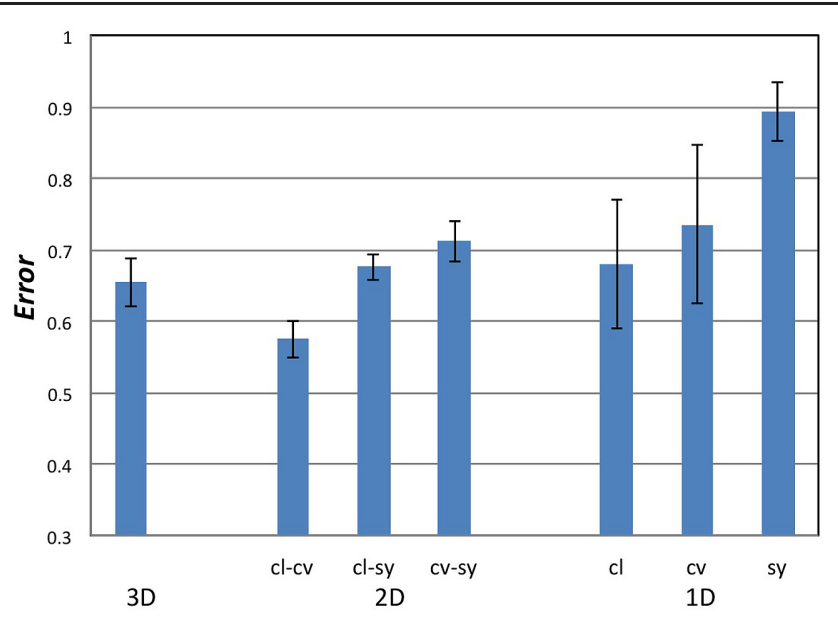

FIGURE 5 | The magnitudes of Error in the MDS analyses. cl, cv, and sy stand for Closure, Convexity, and Symmetry, respectively. The

combination of Closure and Convexity in 2D configuration showed the lowest Error of $57 \%$, indicating that Closure-Convexity accounts for $43 \%$ of the similarity among the local contours.
TABLE 1 | The results of multidimensional scaling (MDS; Experiment 1) for the 1D to 3D configurations in which Convexity, Closure, and Symmetry were topographical factors.

\begin{tabular}{lll}
\hline 1 dimension & $\mathbf{2}$ dimensions & 3 dimensions \\
\hline Convexity 3/6 & $\begin{array}{l}\text { Convexity and } \\
\text { Closure 6/6 }\end{array}$ & $\begin{array}{l}\text { Convexity and Closure and Symmetry } \\
6 / 6 \text { participants were significant }\end{array}$ \\
Closure 5/6 & $\begin{array}{l}\text { Closure and } \\
\text { Symmetry 6/6 }\end{array}$ & \\
Symmetry 3/6 & Closure and & \\
& Convexity 6/6
\end{tabular}

The table shows the number of participants whose perceptual configuration was significantly different $(p<0.05)$ from the random configurations. All participants showed significance in all $2 D$ and $3 D$ combinations.

combination of Closure-Convexity showed the lowest mean Error of $57 \%$, indicating that Closure-Convexity accounts for about $43 \%$ of the similarity. The mean Errors for Closure-Symmetry and Convex-Symmetry were 68 and $71 \%$, respectively, indicating that Closure-Symmetry and Convex-Symmetry account for about 32 and $29 \%$ of the similarity, respectively. The contributions of these two combinations were significantly smaller than that of Closure-Convexity (pair-wise $t$-test, $p<0.01$ ). This result suggests that Closure and Convexity work independently in some cases, although these factors are moderately dependent in topography [Pearson correlation: $\gamma=0.42(p<0.01)]$. The mean Error for the 3D configuration was $65 \%$, indicating that the combination of the three factors account for $35 \%$ of the similarity. Although this contribution is lower than that of Closure-Convexity in 2D (43\%; pair-wise $t$-test, $p<0.01$ ), two participants showed greater contributions in the $3 \mathrm{D}$ case compared to the $1 \mathrm{D}$ and $2 \mathrm{D}$ cases. The combination of the three factors contributes less than a half of the perceptual similarity, suggesting the involvement of other factors in the perception of contour segments. This result appears natural because contour similarity may also be judged by other factors besides the three factors examined here. These results suggest that Convexity, Closure, and Symmetry are perceptual quantities that represent the local shapes of natural contours. Closure and Convexity showed greater contribution than Symmetry in the representation of similarity with an indication of being independent from each other. It should be noticed that the correlation between Symmetry and the perception was lower than that for Convexity and Closure. Although a quantity that better describes the perception of symmetry for arbitrary contours has not been proposed, a further study with such quantity will further reveal the contribution of symmetry.

\section{FIGURE-GROUND SEGREGATION IN LOCAL CONTOURS - EXPERIMENT 2}

We examined whether convexity, closure, and symmetry contribute to local figure-ground segregation in natural contours. We performed psychophysical experiments to judge the direction of the figure along natural contours, and examined the contribution of these factors to the figure-ground judgment. 


\section{Procedure}

We presented the selected set of 105 patches of natural contours (see Materials and Methods), and measured the direction of the figure along the contours. The experimental procedure is shown in Figure 6. We divided the set of patches into two groups, vertical and horizontal groups, depending on the orientation of the contour $\left(0 \pm 45^{\circ}\right.$ and $90 \pm 45^{\circ}$ for vertical and horizontal groups, respectively). Patches from either group were presented for a single session. A single stimulus patch of $4^{\circ} \times 4^{\circ}$ in visual angle was presented at the center of the display, following the presentation of a mask for $1500 \mathrm{~ms}$. A fixation aid of a small red square was presented with the mask. Participants were asked to judge the direction of the figure at the patch center, the location of the fixation aid, by pressing an assigned key in a two-alternative forced-choice paradigm. Specifically, participants were asked to answer right or left for the vertical group, and top or bottom for the horizontal group. Immediately after the response, a mask was presented, and the next trial was begun. All patches were shown in random order with the repetition of 100 . Six participants aged in

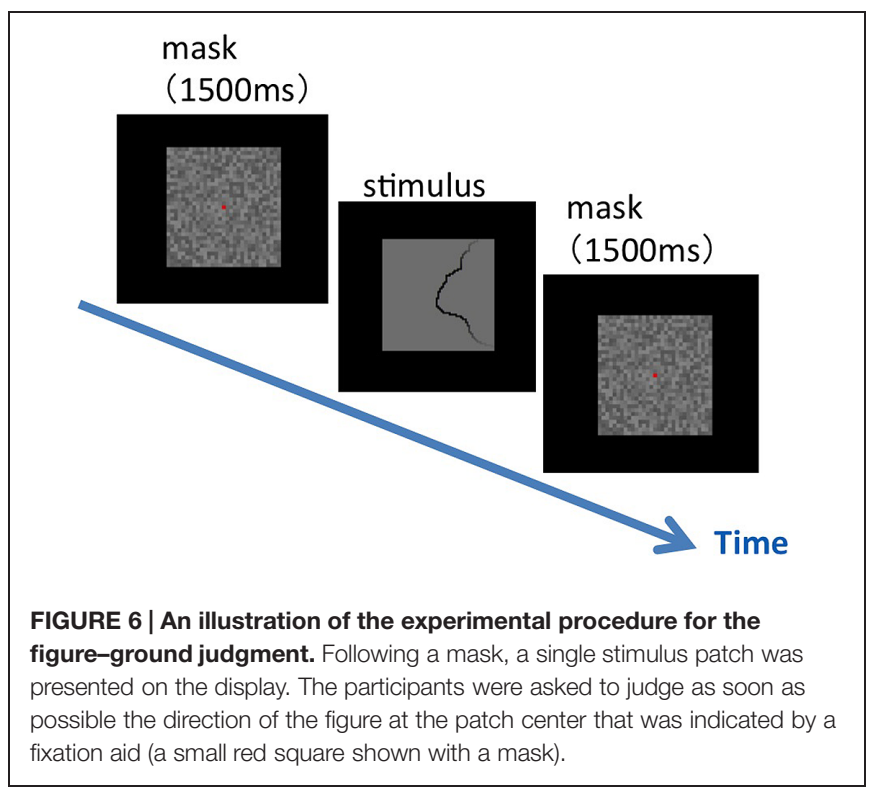

their twenties (21-28) with normal or corrected-to-normal vision underwent the experiment.

\section{Results}

We plotted the rate of choosing a convex side as a function of the indices, Convexity, Closure, and Symmetry. The overall data from all participants are shown in Figure 7. The result shows that the responses depend on Closure (Figure 7B) but not on Convex and Symmetry (Figures 7A,C). We plotted the ratio of judgment in which the participants perceived figure on the convex side, thus a positive Closure means that the convex and closed side was chosen, and a negative means the concave and closed side. Therefore the results indicate that a closed side was perceived as a figure independent of convexity (Figure 7B). A MLRA showed that Closure is significant, but Convexity, Symmetry, and their interactions are not, as shown by the results summarized in Table 2. The adjusted coefficient of determination, $R^{2}$ was 0.54 , indicating a reasonable fit. These results indicate that closure is dominant among the three factors for figure-ground perception in local contours when convexity, closure, and symmetry coexist with equal probability including contradictory cases.

TABLE 2 | The results of multiple linear regression analysis (MLRA) in Experiment 2.

\begin{tabular}{lcc}
\hline & Regression coefficient & $\boldsymbol{p}$-value \\
\hline (Intercept) & 0.490 & $<2 \mathrm{e}-16^{* * *}$ \\
Convexity & -0.334 & 0.262 \\
Closure & 0.0755 & $6.14 \mathrm{e}-13^{* * *}$ \\
Symmetry & 0.0383 & 0.446 \\
Convexity $\times$ Closure & -0.0200 & 0.772 \\
Convexity $\times$ Symmetry & 0.282 & 0.510 \\
Closure $\times$ Symmetry & 0.00557 & 0.536
\end{tabular}

Adjusted $R^{2}: 0.537$

Closure was significant, but Convexity, Symmetry, or interactions were not. The adjusted coefficient of determination, $R^{2}$ was 0.54 , indicating a reasonable fit. The result indicates that closure is a dominant cue for figure-ground perception in natural contours. ${ }^{* * *} p<0.01$.

The model:

(Rate of BO on Convex side) $=a+b \cdot$ Convexity $+c \cdot$ Closure $+d \cdot$ Symmetry + $e \cdot$ Conv $\times$ Clos $+f \cdot$ Conv $\times$ Sym $+g \cdot$ Clos $\times$ Sym

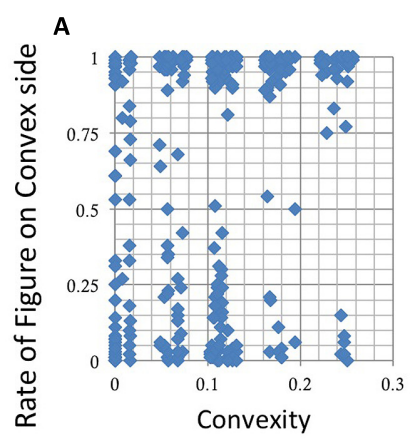

B

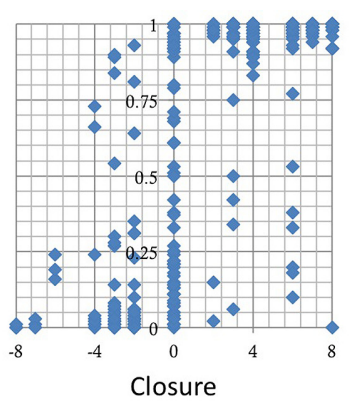

C

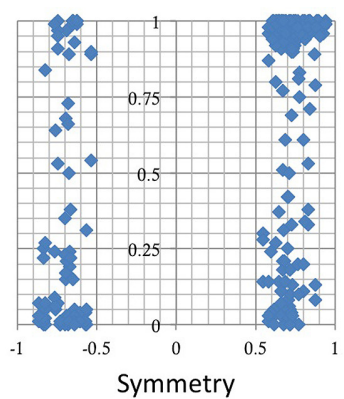

FIGURE 7 | The results of the figure-ground judgment. The rate of choosing a convex side as figure is shown as a function of Convexity (A), Closure (B), and Symmetry (C). The responses depend on Closure but not on Convexity or Symmetry. 
The present result did not show significance in symmetry. A possible bias could be evoked by fixation. We instructed participants to fixate at the patch center where the contour (that defines closure and convexity) always presented while the symmetry axis might not. To examine this possibility, we conducted the same procedure without the fixation. We observed no significant difference from the results with the fixation presented above, indicating no effect of the fixation.

\section{DISCUSSION}

We investigated the perceptual representation of local contour shapes and the contribution of them to local figure-ground segregation. We quantified contour shapes by local cues: convexity, closure, and symmetry. To ensure a wide variety of contour shapes, we sampled patches from natural scenes so that they distributed uniformly over the space that was composed of the three factors. First, we investigated whether the three factors are indeed perceptual quantities that represent the contour shapes. We performed similarity tests between the contour patches, and examined the contribution of the factors to the perceptual similarity between the natural contours. MDS analyses showed that combinations of convexity, closure, and symmetry contribute to the similarity, and thus these factors are indeed perceptual quantities. Second, we examined whether the three factors contribute to the perception of local figureground. We performed psychophysical experiments in which participants judged the direction of the figure along the natural contours, and examined the contribution of the factors to the figure-ground judgment. MLRA showed that closure reached significance, but convexity and symmetry did not. These results indicate that closure is a dominant cue for local figure-ground perception when convexity, closure, and symmetry coexist with equal probability including contradictory cases.

Convexity has long been known as a strong cue for figureground perception. Combinations with other cues have also been studied, including the comparison with symmetry (e.g., Kanizsa and Gerbino, 1976; Devinck and Spillmann, 2013; Mojica and Peterson, 2014), parallelism (Froyen et al., 2013), and top-down effects (Devinck and Spillmann, 2013). These studies showed relative strength of convexity compared with the other cues. For instance, convexity evoked stronger 3D volume from structure-from-motion compared with symmetry and parallelism. Convexity evoked shorter reaction times for detecting figures in ambiguous boundaries compared with symmetry and top-down effects. However, these studies presented multiple, artificial contours with simple and regular shapes in which two cues were contradictory. Our experiments were distinct from these previous studies in stimuli; (1) ours consisted of a single contour in a number of cases, and (2) our stimuli were controlled natural contours in which multiple cues coexist with a wide range of relative strengths from consistent to contradictory cases. Fowlkes et al. (2007) used natural contours to test the relative strength of convexity, relative size, and lower region, and reported that convexity was weakest among the three cues. Natural contours in which multiple cues are inherent in a single contour may work differently from pairs of artificial contours with contradictory cues.

Although our results did not indicate the significance of convexity, our result may be consistent with previous reports. Gestalt factors are not necessarily independent. Convexity and closure appear to be often dependent on each other; in fact, the coefficient of Pearson correlation between Convexity and Closure was $0.42(p<0.01)$ in our stimulus set. A closed contour within a stimulus patch has a large degree of convexity. Thus, the essence of convexity can be considered closedness. From an ecological viewpoint, a closed contour is a strong cue for an object (figure). If the object is occluded and only some parts of the object are visible, convexity may be a good cue to substitute for closure. Convexity and closure can also be independent. As shown in the right panels of Figure 1C, a closed region may have a concave contour. Our result showed that in such a case, closure is dominant over convexity in figureground perception. Because we designed the experiments to have a wide and uniform distribution of the indices of the stimulus set, the number of the independent cases was close to that of the dependent cases. This uniform distribution is different from the natural distribution in which the dependent cases are more frequent. The uniform distribution appears to successfully separate the indices. If a stimulus set had the natural distribution, a greater number of dependent cases might conceal the independent cases and result in a failure to separate the indices.

Our results did not indicate the significance of symmetry in the figure-ground judgment. Symmetry has long been known as a strong cue for the perception of an object, and also as a basis for the neural representation of shape (e.g., Hung et al., 2012; Hatori and Sakai, 2014). The adaptation to symmetry has recently reported, indicating that symmetry is a perceptual quantity (Gheorghiu et al., 2014; Sakai et al., 2014). A crucial difference between our experiment and the previous studies is that our stimuli were local contours that consisted mostly of a single open contour. Thus, our results suggest that symmetry may not be as influential as closure with an open local contour. Symmetry may be crucial from an ecological viewpoint, such as in the detection of a living being that often has a closed contour.

\section{ACKNOWLEDGMENT}

This work was supported by a grant-in-aid from JSPS (KAKENHI 26280047), grant-in-aid of Scientific Research on Innovative Areas, "Shitsukan" (No. 25135704) from MEXT, Japan, and a grant-in-aid from RIEC, Tohoku University (H25/A09).

\section{SUPPLEMENTARY MATERIAL}

The Supplementary Material for this article can be found online at: http://journal.frontiersin.org/article/10.3389/fpsyg. 2015.01685 


\section{REFERENCES}

Altmann, C. F., Bülthoff, H. H., and Kourtzi, Z. (2003). Perceptual organization of local elements into global shapes in the human visual cortex. Curr. Biol. 13, 342-349. doi: 10.1016/S0960-9822(03)00052-6

Borg, I., and Groenen, P. (1997). Modern Multidimensional Scaling: Theory and Applications. New York, NY: Springer.

Brunswik, E., and Kamiya, J. (1953). Ecological cue-validity of "proximity" and of other gestalt factors. Am. J. Psychol. 66, 20-32. doi: 10.2307/1417965

Burge, J., Fowlkes, C. C., and Banks, M. S. (2010). Natural-scene statistics predict how the figure-ground cue of convexity affects human depth perception. J. Neurosci. 30, 7269-7280. doi: 10.1523/JNEUROSCI.5551-09.2010

Cohen, E. H., and Zaidi, Q. (2013). Symmetry in context: salience of mirror symmetry in natural patterns. J. Vis. 13:22. doi: 10.1167/13.6.22

Devinck, F., and Spillmann, L. (2013). Multiple cues add up in defining a figure on a ground. Vis. Res. 77, 51-58. doi: 10.1016/j.visres.2012.10.021

Dillon, W. R., and Goldstein, M. (1984). Multivariate Analysis. New York, NY: John Wiley \& Sons.

Elder, J. H., and Zucker, S. W. (1998). Evidence for boundary-specific grouping. Vis. Res. 38, 143-152. doi: 10.1016/S0042-6989(97)00138-7

Fowlkes, C. C., Martin, D. R., and Malik, J. (2007). Local figure-ground cues are valid for natural images. J. Vis. 72, 1-14.

Froyen, V., Feldman, J., and Singh, M. (2013). Rotating columns: relating structure-from-motion, accretion/deletion, and figure/ground. J. Vis. 13:6. doi: $10.1167 / 13.10 .6$

Gheorghiu, E., Bell, J., and Kingdom, F. A. A. (2014). Visual adaptation to symmetry. J. Vis. 4:63. doi: 10.1167/14.10.63

Gilad, A., Pesoa, Y., Ayzenshtat, I., and Slovin, H. (2014). Figure-ground processing during fixation saccades in V1: indication for high-order stability. J. Neurosci. 34, 3247-3252. doi: 10.1523/JNEUROSCI.4375-13.2014

Hatori, Y., and Sakai, K. (2014). Early representation of shape by onset synchronization of border-ownership-selective cells in the V1-V2 network. J. Opt. Soc. Am. A Opt. Image Sci. Vis. 31, 716-729. doi: 10.1364/JOSAA.31.000716

Hung, C. C., Carlson, E. T., and Connor, C. E. (2012). Medial axis shape coding in macaque inferotemporal cortex. Neuron 74, 1099-1113. doi: 10.1016/j.neuron.2012.04.029

Hurley, J. R., and Cattell, R. B. (1962). The Procrustes program: producing direct rotation to test a hypothesized factor structure. Behav. Sci. 7, 258-262. doi: 10.1002/bs.3830070216

Ito, M., and Komatsu, H. (2004). Representation of angles embedded within contour stimuli in area V2 of macaque monkeys. J. Neurosci. 24, 3313-3324. doi: 10.1523/JNEUROSCI.4364-03.2004

Kanizsa, G. (1979). Organization in Vision: Essays on Gestalt Perception. New York, NY: Praeger

Kanizsa, G., and Gerbino, W. (1976). "Convexity and symmetry in figure-ground organization," in Vision and Artifact, ed. M. Henle (New York, NY: Springer).

Kim, S. H., and Feldman, J. (2009). Globally inconsistent figure/ground relations induced by a negative part. J. Vis. 9, 1-13. doi: 10.1167/9.10.8

Kimchi, R., Hadad, B., Behrmann, M., and Palmer, S. E. (2005). Microgenesis and ontogenesis of perceptual organization. Psychol. Sci. 16, 282-290. doi: 10.1111/j.0956-7976.2005.01529.x

Kimchi, R., and Peterson, M. A. (2008). Figure-ground segregation can occur without attention. Psychol. Sci. 19, 660-668. doi: 10.1111/j.14679280.2008.02140.x

Kourtzi, Z., Tolias, A. S., Altmann, C. F., Augath, M., and Logothetis, N. K. (2003). Integration of local features into global shapes: monkey and human fMRI studies. Neuron 37, 333-346. doi: 10.1016/S0896-6273(02)01174-1

Kovács, I. (1996). Gestalten of today: early processing of visual contours and surfaces. Behav. Brain Res. 82, 1-11. doi: 10.1016/S0166-4328(97)81103-5
Kruscal, J. B. (1964). Multidimensional scaling by optimizing goodness of fit to a nonmetric hypothesis. Psychometrika 29, 1-27. doi: 10.1007/BF02289565

Martin, D., Fowlkes, C. C., Tal, D., and Malik, J. (2001). "A database of human segmented natural images and its application to evaluating segmentation algorithms and measuring ecological statistics," in Proceedings of the Eighth IEEE International Conference on Computer Vision, Vol. 2 (Los Alamitos, CA: IEEE Computer Society), 416-425.

Mojica, A. J., and Peterson, M. A. (2014). Display-wide influences on figureground perception: the case of symmetry. Attent. Percept. Psychophys. 76, 1069-1084. doi: 10.3758/s13414-014-0646-y

Pasupathy, A., and Connor, C. E. (1999). Responses to contour features in macaque area V4. J. Neurophysiol. 82, 2490-2502.

Rainville, S. J. M., and Kingdom, F. A. A. (2000). The functional roles of oriented spatial filters in the perception of mirror symmetry - psychophysics and modeling. Vis. Res. 40, 2621-2644. doi: 10.1016/S0042-6989(00)0 0110-3

Sajda, P., and Finkel, L. H. (1995). Intermediate-level visual representation and the construction of surface perception. J. Cogn. Neurosci. 7, 267-291. doi: 10.1162/jocn.1995.7.2.267

Sakai, K., Kurematsu, K., and Matuoka, S. (2014). "Perception of symmetry in natural images a cortical representation of shape," Neural Information Processing, Vol. 8836, LNCS, eds C. K. Loo, K. S. Yap, K. W. Wong, A. T. Beng Jin, and K. Huang (Cham: Springer), 135-141.

Sakai, K., and Michii, S. (2013). "Latency modulation of border ownership selective cells in V1-V2 feed-forward model," in Neural Information Processing, Vol. 8228, LNCS, eds M. Lee, A. Hirose, Z.-H. Hou, and R. M. Kil (Berlin: Springer), 291-300.

Sakai, K., and Nishimura, H. (2006). Surrounding suppression and facilitation in the determination of border ownership. J. Cogn. Neurosci. 18, 562-579. doi 10.1162/jocn.2006.18.4.562

Sakai, K., Nishimura, H., Shimizu, R., and Kondo, K. (2012). Consistent and robust determination of border ownership based on asymmetric surrounding contrast. Neural Netw. 33, 257-274. doi: 10.1016/j.neunet.2012.05.006

Sasaki, Y., Vanduffel, W., Knutsen, T., Tyler, C., and Tootell, R. (2005). Symmetry activates extrastriate visual cortex in human and nonhuman primates. Proc. Natl. Acad. Sci. U.S.A. 102, 3159-3163. doi: 10.1073/pnas.050031 9102

Super, H., Romeo, A., and Keil, M. (2010). Feed-forward segmentation of figure-ground and assignment of border-ownership. PLOS ONE 5:e10705. doi: 10.1371/journal.pone.0010705

Tyler, C. W., Baseler, H. A., Kontsevich, L. L., Likova, L. T., Wade, A. R., and Wandell, B. A. (2005). Predominatly extra-retinotopic cortical response to pattern symmetry. Neuroimage 24, 306-314. doi: 10.1016/j.neuroimage.2004.09.018

Zhang, N. R., and von der Heydt, R. (2010). Analysis of the context integration mechanisms underlying figure-ground organization in the visual cortex. J. Neurosci. 30, 6482-6496. doi: 10.1523/JNEUROSCI.5168-09.2010

Zhou, H., Friedman, H. S., and von der Heydt, R. (2000). Coding of border ownership in monkey visual cortex. J. Neurosci. 20, 6594-6611.

Conflict of Interest Statement: The authors declare that the research was conducted in the absence of any commercial or financial relationships that could be construed as a potential conflict of interest.

Copyright (c) 2015 Sakai, Matsuoka, Kurematsu and Hatori. This is an open-access article distributed under the terms of the Creative Commons Attribution License (CC BY). The use, distribution or reproduction in other forums is permitted, provided the original author(s) or licensor are credited and that the original publication in this journal is cited, in accordance with accepted academic practice. No use, distribution or reproduction is permitted which does not comply with these terms. 